Enferm Bras 2019;18(2):264-72

https://doi.org/10.33233/eb.v18i2.2469

\title{
ARTIGO ORIGINAL \\ Distribuição espacial e temporal da incidência da hanseníase em menores de 15 anos em Manaus
}

Marcos Vinícius Costa Fernandes*, Arinte Véras Fontes Esteves**, Cláudia Benetida dos Santos $^{* \star *}$, Daniel Barros de Castro***

*Universidade do Estado do Pará e Universidade Federal do Amazonas, Programa de Pósgraduação em Enfermagem, **Universidade do Estado do Pará e Universidade Federal do Amazonas, Programa de Pós-graduação em Enfermagem, Manaus/AM, ${ }^{* *}$ Universidade de São Paulo, Programa Nacional de Cooperação Acadêmica, Escola de Enfermagem de Ribeirão Preto,

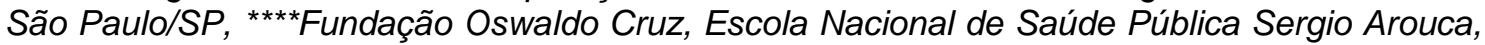
Rio de Janeiro/RJ

Recebido em 25 de julho de 2018; aceito em 12 de dezembro de 2018.

Endereço de correspondência: Marcos Vinícius Costa Fernandes, Rua Teresina, 495, Adrianópolis, Manaus AM, E-mail: mvcf_2012@hotmail.com; Arinte Véras Fontes Esteves: arineteveras@ig.com.br; Cláudia Benetida dos Santos: cbsantos@eerp.usp.br; Daniel Barros de Castro: danielbarrosbio@gmail.com

\begin{abstract}
Resumo
Introdução: A hanseníase é uma doença milenar, permanecendo no século XXI como um grande desafio à saúde pública mundial. Objetivo: Analisar a distribuição espacial e temporal da incidência de hanseníase em menores de 15 anos no município de Manaus, no período de 2009 a 2015. Métodos: Estudo epidemiológico de caráter descritivo do tipo ecológico, com unidade de análise dos bairros. Os dados foram obtidos no Sistema de Informação de Agravos de Notificações. As unidades de análises foram classificadas de acordo com o grau de endemicidade. Foram calculadas as taxas de incidência para cada ano; em seguida, para analisar a distribuição espacial e temporal foram construídos mapas temáticos, com o software QGIS. Resultados: No período de 2009 a 2015 foram notificados 173 casos novos de hanseníase em menores de 15 anos em Manaus. Os indicadores apresentaram um gradiente, na distribuição por zonas, apontando cinco áreas onde se concentram bairros com taxas de detecção classificados entre média e elevada incidência, que são os bairros das regiões Norte, Sul, Leste, Centro-sul. Conclusão: O conhecimento detalhado do município de Manaus, observando o processo saúde-doença da hanseníase em menores de 15 anos, descreveu a magnitude e distribuição desse problema de saúde pública.
\end{abstract}

Palavras-chave: criança, hanseníase, epidemiologia.

\begin{abstract}
Spatial and temporal distribution of incidence of leprosy in children under 15 years of age in Manaus

Introduction: Leprosy is a millennial disease, remaining in the 21 st century as a major challenge to global public health. Objective: To analyze spatial and temporal distribution of the incidence rates of leprosy in children under 15 years in Manaus, from 2009 to 2015. Methods: This is an epidemiological study of a descriptive approach and ecological type, with analysis of the neighborhoods. Data were obtained from the Notifiable Diseases Information System. The units of analysis were classified according to the degree of endemicity. The incidence rates were calculated for each year; then to analyze the spatial and temporal distribution were constructed thematic maps, with the QGIS software. Results: In the period from 2009 to 2015,173 new cases of leprosy were reported in children less than 15 years in Manaus. The indicators presented a gradient, in the distribution by zones, pointing out five areas where districts with detection rates are concentrated and classified as medium and high incidence, which are the districts of the North, South, East, Center-South regions. Conclusion: The detailed knowledge of the municipality of Manaus, observing the health-disease process of leprosy in children under 15, described the magnitude and distribution of this public health problem.
\end{abstract}


Key-words: child, leprosy, epidemiology.

\begin{abstract}
Resumen
Distribución espacial y temporal de la incidencia de la lepra en menores de 15 años en Manaus

Introducción: La hanseniasis es una enfermedad milenaria, permaneciendo en el siglo XXI como un gran desafío a la salud pública mundial. Objetivo: Analizar la distribución espacial y temporal de la incidencia de hanseniasis en menores de 15 años en el municipio de Manaus, en el período de 2009 a 2015. Métodos: Estudio epidemiológico de carácter descriptivo del tipo ecológico, con unidad de análisis los barrios. Los datos fueron obtenidos de las bases de datos del Sistema de Información de Agravamientos de Notificaciones. Las unidades de análisis se clasificaron de acuerdo con el grado de endemicidad. Se calcularon las tasas de incidencia para cada año; a continuación, para analizar la distribución espacial y temporal se construyeron mapas temáticos, con el software QGIS. Resultados: En el período de 2009 a 2015 se notificaron 173 casos nuevos de lepra en menores de 15 años en Manaus. Se ha producido una caída del número de casos nuevos del año 2009 a 2015, sin embargo el recorrido temporal de la enfermedad presenta una oscilación entre el declive y el aumento de los casos de 2009 a 2013. Resultados: Los indicadores presentaron un gradiente, en la distribución por zonas, apuntando cinco áreas donde se concentran barrios con tasas de detección clasificados entre media y alta incidencia, que son los barrios de las regiones Norte, Sur, Este, Centro-sur. Conclusión: El conocimiento detallado del municipio de Manaus, observando el proceso salud-enfermedad de la lepra en menores de 15 años, describió la magnitud y distribución de ese problema de salud pública.
\end{abstract}

Palabras-clave: niño, lepra, epidemiología.

Introdução

A hanseníase é uma doença milenar, permanecendo no século XXI como um grande desafio à saúde pública mundial [1]. Dados referenciados de 2016 publicados pela Organização Mundial de Saúde (OMS) mostram que 145 países apresentaram registro de 214.783 novos casos de hanseníase. No Brasil foi evidenciado em 2016 um coeficiente de detecção geral de 12,23 por 100 mil hab. e o coeficiente de detecção em menores de 15 anos de 3,63 por 100 mil hab. Com todo avanço em 2016, o Brasil não atingiu a meta estabelecida aos países membros da OMS, visto que apresentam mais de um caso para cada 100.000 habitantes em menores de 15 anos, sendo assim considerado um país endêmico [2,3].

No Amazonas, a situação de hanseníase é preocupante, com 186 casos, destes 144 novas infecções, 23 recidivas, 14 reingressos e 07 transferências. Na detecção de novos casos, 12 foram em menores de 15 anos. A detecção na população pediátrica sinaliza uma deficiência no controle e vigilância da doença pelos gestores de saúde, pois uma criança com hanseníase significa que alguém do convívio dela também tem a doença e não está realizando o tratamento [4].

Um estudo realizado em Manaus para analisar a distribuição espacial dos casos novos de hanseníase em menores de 15 anos e as condições de vida no período de 1998 a 2004 enfatizou que a utilização da análise espacial pôde auxiliar na compreensão da distribuição da doença no município, contribuindo para elucidação da sua relação com questões socioeconômicas e crescimento urbano, confirmando a gravidade do nível de endemia da hanseníase e sinalizando as áreas prioritárias para a intervenção de políticas públicas de saúde [5].

Ainda no que se refere à distribuição espacial de hanseníase em Manaus, um estudo entre os anos de 1998 a 2009, com o objetivo de conhecer o perfil do custo das doenças fortemente atribuíveis a fatores ambientais na cidade de Manaus, observou o padrão de distribuição espacial do número de casos novos diagnosticados na população geral e sua relação com o padrão de expansão urbana em Manaus após a instalação da Zona Franca (1967), que trouxe novas áreas de ocupação regular e irregulares [6].

No presente artigo, busca-se analisar a distribuição espacial dos casos novos de hanseníase em menores de 15 anos. 
Este artigo buscou realizar um estudo descritivo do tipo ecológico misto, cuja unidade de análise foi os bairros de Manaus. O município está localizado na região Norte do Brasil, situado a 0306'07' S de latitude e 6001'30" W de longitude, possui área territorial de $11.401,092$ km². Em 2010 sua população era de 1.802 .014 habitantes e com uma densidade demográfica de 158,06 habitantes $/ \mathrm{km}^{2}$ [7].

A população de estudo constituiu-se dos menores de 15 anos residentes em Manaus, diagnosticados com hanseníase no período de 2009 a 2015, notificados no Sistema Nacional de Agravos de Notificação (SINAN).

Foram excluídos os registros duplicados e aqueles em que os campos "idade", "bairro de residência", "modo de entrada" e "tipo de alta" estavam em branco ou preenchido com "ignorado", além daqueles cujos registros descritos como erro de diagnóstico. Foram consideradas duplicações aqueles registros cujo campo data de nascimento, nome do paciente e nome da mãe era igual.

Os dados sobre a população dos bairros foram obtidos a partir do censo realizado em 2000 e 2010 pelo Instituto Brasileiro de Geografia e Estatística (IBGE) e as populações do período intercensitário foram obtidas pelo método de interpolação linear.

A taxa de incidência de hanseníase em menores de 15 anos em cada bairro foi calculada dividindo o total de casos novos em menores de 15 anos de idade residentes em determinado bairro e diagnosticados em cada ano estudado, pelo total da população de zero a 14 anos de idade, no mesmo local e período, multiplicado por 100 mil habitantes.

Após o cálculo da taxa de incidência realizou-se uma análise descritiva por meio de distribuições de frequências, valores médios, medianos e desvio padrão das taxas de incidência. O programa estatístico utilizado foi o Statistical Package for Social Sciences (SPSS, 1999), versão 19. 0 (NFS-e 3076, licença no 10101111255).

Os bairros de Manaus foram classificados segundo o grau de endemicidade, conforme preconizado pelo Ministério da Saúde (MS), como: (i) hiperendêmico: $\geq 10,00$ por 100 mil hab.; (ii) muito alto: 5,00 a 9,99 por 100 mil hab.; (iii) alto: 2,50 a 4,99 por 100 mil hab.; (iv) médio: 0,50 a 2,49 por 100 mil hab.; (v) baixo: < 0,50 por 100 mil hab. [6]. Foram construídos de mapas temáticos sequenciais por meio do software QGIS versão 2.18.16.

Este estudo foi aprovado pelo Comitê de Ética em Pesquisa da Universidade Federal do Amazonas (UFAM), com número do Parecer: 1.860.181 [8].

\section{Resultados}

Foram notificados 2.207 novos casos de hanseníase na população geral no período de 2009 a 2015. Desse total, 173 (9\%) casos ocorreram em menores de 15 anos e depreende-se que 91 notificações (53\%) eram de indivíduos do sexo masculino, havendo predomínio sobre o sexo feminino $(47 \%)$.

Tabela I - Casos novos de hanseníase em adultos segundo o sexo em Manaus no período de 2009 a 2015.

\begin{tabular}{ccccc}
\hline Sexo & $\geq 15$ anos & $\%$ & $<15$ anos & $\%$ \\
\hline Feminino & 632 & 37 & 82 & 47 \\
Masculino & 1.073 & 63 & 91 & 53 \\
\hline Total & 1.705 & 100 & 173 & 100 \\
\hline Fonte: Sistema Nacional de Agravos de Notificação - SINAN.
\end{tabular}

Conforme a Tabela II, verificou-se que o coeficiente de incidência neste grupo etário oscilou, ao longo dos anos entre 2009-2015, de 6,3 casos/100 mil hab. classificado como muito alto e 3,8 casos/100 mil hab. que é considerado alto nível endêmico, de acordo com Ministério da Saúde (MS). Neste mesmo período o coeficiente de incidência anual médio em menores de 15 anos foi de 4,7 casos/100 mil hab. classificado como alto nível endêmico. 
Tabela II - Casos novos de hanseníase em crianças e coeficientes de incidência por 100 mil habitantes em Manaus no período de 2009 a 2015.

\begin{tabular}{lllll}
\hline Ano & Casos novos em crianças & $\begin{array}{l}\text { População total de } \\
\text { menores de } 15 \text { anos } \\
\text { de Manaus }\end{array}$ & $\begin{array}{l}\text { Incidência } \\
(/ 100.000 \\
\text { habitantes })\end{array}$ & Parâmetros \\
\hline 2009 & 27 & 507.582 & 5.3 & Muito alto \\
2010 & 22 & 508.962 & 4.3 & Alto \\
2011 & 26 & 517.551 & 5.0 & Muito alto \\
2012 & 20 & 525.857 & 3.8 & Alto \\
2013 & 33 & 534.163 & 6,3 & Muito alto \\
2014 & 24 & 542.469 & 4.6 & Alto \\
2015 & 21 & 550.775 & 4.0 & Alto \\
\hline Total & 173 & 3.687 .359 & 4.7 & Alto \\
\hline
\end{tabular}

Fonte: Sistema Nacional de Agravos de Notificação - SINAN; População: IBGE - Censos Demográficos e Contagem Populacional (2010).

Verificou-se uma distribuição heterogênea quando analisada a evolução da taxa de incidência de hanseníase no período estudado, havendo uma variação entre a redução e aumento no período estudado. Evidencia-se ainda que os bairros de Manaus mantiveram um padrão heterogênico em suas taxas de incidência em relação a novos casos de hanseníase. Foi observado um aglomerado de bairros com elevada incidência de hanseníase nas regiões Norte, Sul, Leste e Centro-sul, e em poucos bairros da região Oeste do município de Manaus (Figura 1).

A análise da distribuição do número de casos novos de hanseníase em menores de 15 anos aponta que o número de casos novos se apresentou com oscilação nos padrões temporais. Observou-se que houve queda do número de casos novos do ano de 2009 a 2015, entretanto o percurso temporal da doença apresenta uma oscilação entre o declínio e aumento dos casos da hanseníase na cidade de Manaus no período de 2009 a 2013. 

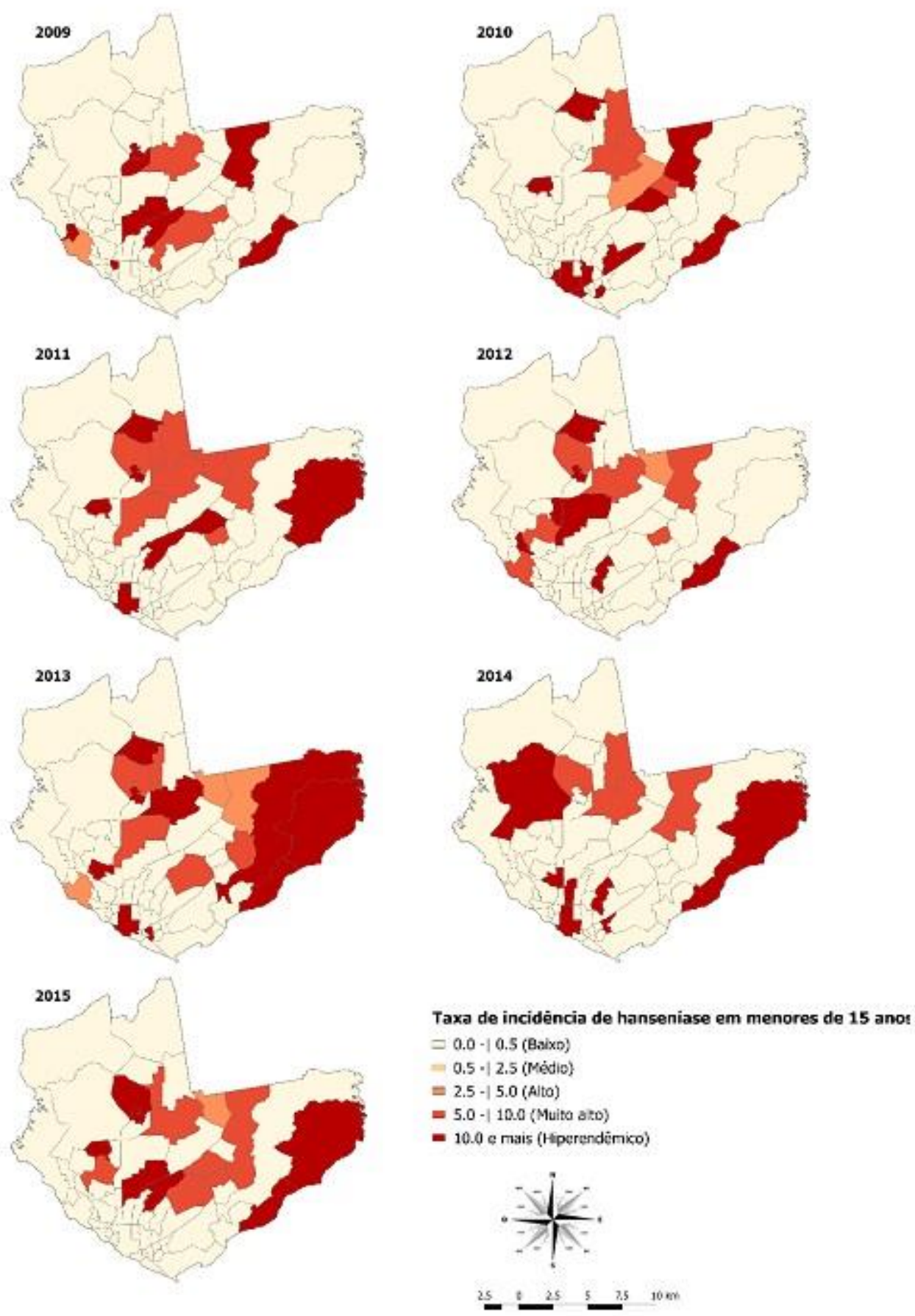

Fonte: Sistema Nacional de Agravos de Notificação - SINAN; IBGE - Censos Demográficos e Contagem Populacional (2010).

Figura 1 - Distribuição espacial da taxa de incidência de hanseníase em menores de 15 anos nos bairros de Manaus (mapas sequenciais).

\section{Discussão}

Através do presente estudo pode-se identificar a predominância dos casos de hanseníase em indivíduos do sexo masculino. Este perfil epidemiológico da hanseníase corrobora demais estudos feitos na Amazônia e em outros Estados do Brasil, os quais afirmam a prevalência da doença no sexo masculino em relação ao feminino no período de 2009 a 2015 [9-11].

Relacionada a fatores com a identidade masculina, o maior contato interpessoal no trabalho, menor preocupação com seu bem-estar físico e a falta de acesso aos serviços médicos 
torna esse grupo mais propicio a doenças infeciosas e outros agravos. O que sugere a hipótese que as condições de vida mais ativa do homem e o descaso com a saúde os torna mais expostos à doença $[12,13]$.

Com relação à hanseníase em menores de 15 anos em Manaus, que houve transmissão ativa nesta faixa etária, no período de 2009 a 2015, é valido ressaltar que a presença de casos na população pediátrica é um indicador da gravidade do nível endêmico da hanseníase e reflete a exposição precoce de menores ao bacilo, uma vez que este agravo não é considerado uma doença prevalente na infância [5].

Sabe-se que onde há adultos com a doença sem tratamento seja vizinho ou familiar contribuem para o destaque epidemiológico em menores de 15 anos, devido à exposição precoce ao bacilo. Sendo assim, a infecção em menores de 15 anos é mais frequente quando existem casos na família e em áreas de incidência elevada da doença, tornado assim a vigilância de casos neste grupo como princípio fundamental para a erradicação da hanseníase [14].

Essa afirmativa foi demonstrada por estudos realizados nos Estados do Mato Grosso e Minas Gerais que revelaram a exposição precoce de crianças ao bacilo, em decorrência do contato íntimo e prolongado com os familiares bacilíferos não tratados somado ao agravante da defesa imunológica deficitária na infância. Sinalizando ainda que a prevalência de casos em crianças pode ser um indicador de um sistema de saúde ineficiente no controle e tratamento de casos na população adulta e a busca de casos insatisfatória em menores de 15 anos, permanecendo assim focos de transmissão ativos da hanseníase [14,15].

Destaca-se, na literatura, um caso raro de diagnóstico clínico de hanseníase multibacilar, sendo a forma mais grave da doença, em uma criança de três anos de idade corroborando a exposição precoce da criança ao bacilo. Esta forma da doença favorece a apresentação de incapacidade física e é menos frequente em crianças devido ao seu longo período de incubação. Em síntese, a detecção e tratamento precoce da hanseníase na população geral quebra sua cadeia de transmissão e previne a incidência de casos novos na infância. Além disto, a presença de casos nesta população jovem é um indicativo da deficiência nos serviços de saúde quanto à detecção precoce da doença [16].

Em Manaus, o coeficiente de detecção de casos novos de hanseníase em menores de 15 anos variou no período estudado de muito alto e alto nível endêmico, mostrando uma tendência de redução. O declínio da taxa da hanseníase ao longo dos anos pode estar relacionado à implantação de estratégias governamentais com o intuito de promover saúde e prevenir doenças e agravos. Umas das estratégias foi o Programa Saúde na Escola (PSE) com o objetivo de promover a atenção integral à saúde da criança, adolescente e adultos, por meio da educação em saúde, fortalecendo, assim, os espaços de participação com discentes, docentes e profissionais de saúde para a construção da educação em saúde [17].

O PSE prevê, por meio da parceria entre o MS e Ministério da Educação (ME), conscientizar o autoexame e realizar a busca ativa de casos entre escolares de forma articulada com o Plano integrado de ações estratégicas de eliminação de doenças negligenciadas e relacionadas à pobreza, otimizando, assim, o compromisso nacional de redução da carga da hanseníase nos grupos populacionais mais vulneráveis situados em áreas geográficas de maior risco [17-19].

Apesar dos esforços do governo, em relação à criação de políticas públicas buscando a promoção da saúde e prevenção de doenças, Manaus apresenta um coeficiente de incidência acima da média proposta pelo MS. Os resultados encontrados na pesquisa corroboram outro estudo realizado no Brasil, que enfatiza que a hanseníase em menores de 15 anos ainda é um alerta para os gestores de saúde e a participação da atenção primária baseada na Estratégia da Saúde da Família desenvolvendo atividades de educação em saúde representa uma forma eficaz na prevenção de doenças endêmicas [20,21].

Em Manaus os profissionais de saúde responsáveis pela promoção da saúde e prevenção de agravos se encontram nos seus quatro Distritos de Saúde (DISA): Distrito Sul, Distrito Norte, Distrito Oeste, Distrito Leste. Neste contexto a atenção primaria à saúde deve atuar nos territórios de sua abrangência, proporcionando atenção integral a população. É válido ressaltar que as condições de trabalho das equipes de Saúde da Família são determinantes para o seu processo de trabalho. Sendo assim, a falta de materiais de insumo e infraestrutura inadequada das Unidades Básicas de Saúde pode colaborar com a fragilidade no serviço de saúde em áreas que muitas vezes são marcadas pelas condições de vulnerabilidade socioeconômicas [21].

Outros estudos no país apresentaram alta endemicidade da doença, em Salvador a distribuição da magnitude das taxas anuais de casos em menores de 15 anos apresentou-se em 
endemicidade muito alta (5,4 casos/100 mil hab.), além de detectar casos em menores de 4 anos $(10,3 \%)$ sendo mais um fato importante para a evidência da transmissão ativa da doença, este fato de contágio reflete a inoperância das políticas públicas de saúde. Conforme já mostrado na literatura, é válido ressaltar que a meta da Estratégia Global para Hanseníase 2016-2020 é de reduzir a zero o número de crianças diagnosticadas com hanseníase $[1,22]$.

Contudo, a Estratégia Global para Hanseníase 2016-2020 ressalta a luta mundial contra a hanseníase, aperfeiçoando e aprimorando as ações conjuntas globais e locais para o diagnóstico e tratamento precoce da doença, redução da incapacidade física e melhorias das condições de vida da população. Embora o Brasil já tenha estabelecido fortalecimento para vigilância, atenção e controle da hanseníase por meio do Programa Nacional de Controle da Hanseníase $(\mathrm{PNCH})$, que desenvolve ações de educação permanente visando melhorar a qualidade da prática assistencial nos diferentes níveis de atenção, além de fortalecer a vigilância epidemiológica desta patologia, o país não conseguiu estabilizar os indicadores epidemiológicos da doença $[2,22]$.

O detalhamento dos bairros de Manaus como unidades de análise permitiu a identificação das áreas de maior endemicidade da doença, o que pode vir a contribuir para o planejamento de ações de controle da hanseníase no município. A taxa de incidência em menores de 15 anos mostra uma elevada exposição à doença nesta faixa etária, particularmente nos bairros das regiões Norte, Sul e Leste.

Tal resultado corrobora estudo realizado em Manaus no ano de 2009, que em sua análise espacial mostrou a hanseníase heterogênea, atingindo intensamente as regiões Norte, Sul e Leste, sua taxa de detecção variou de hiperendêmico e muito alto nos setores de estudo. Em 2011, outra pesquisa apontou alta taxa de incidência de hanseníase nessas mesmas regiões, relacionado à distribuição espacial de casos da doença com o padrão de expansão urbana de Manaus na década de 1990, nas zonas Sul e Oeste e partir de 2000 na Leste e Norte da cidade [21].

A mudança do padrão de expansão urbana em Manaus ocorreu entre o final do século XIX e o começo do século XX, quando a capital se tornou o principal centro financeiro da Região Norte, o que proporcionou a expansão urbana e demográfica da cidade, trazendo novas áreas de ocupação. As zonas Sul, Centro-sul, Oeste e Centro-oeste eram as áreas onde se encontravam os maiores espaços urbanos. Com a implantação da Zona Franca de Manaus, em 1967, houve um êxodo rural, das populações oriundas do interior do estado e de cidade vizinhas em busca de melhores condições de vida, este fato contribuiu para o surgimento de ocupações irregulares e regulares, influenciando a expansão das zonas Leste e Norte [23].

Diante da crítica situação de ocupação irregular em decorrência do aumento populacional e da expansão da cidade, principalmente a partir da criação da Zona Franca de Manaus, pode-se observar o agravamento da saúde pública e a exclusão social na região, como consequência do êxodo rural e a migração de famílias que buscavam uma nova oportunidade de vida, favorecendo ocupações em locais insalubres e com precárias condições de vida por aqueles que não conseguiram se inserir no mercado de trabalho, e se depararam com 0 desemprego e baixa fonte de renda [24].

A caracterização da expansão urbana e demográfica desordenada em Manaus pode explicar a repercussão da dinâmica de problemas de saúde pública nessas zonas administrativas e ser o ponto chave para justificar a distribuição demográfica da hanseníase na cidade. Na zona Sul, sendo umas das zonas mais antiga da cidade, as características sociodemográficas influenciaram no aumento da densidade populacional, além de apresentar os bairros com maior número de população, com construções de moradias precárias, o contato íntimo e prolongado é maior no ambiente domiciliar, o que favorece a transmissão do bacilo [5].

Os impactos ambientais negativos significativos nas zonas Norte e Leste ocorreram devido ao intenso processo de ocupação destas, tendo destaque a zona Leste que apresentou uma forma de ocupação irregular em encostas, nascente de igarapé, barrancos e próximos a rede elétrica de alta tensão, chamadas de invasões. Essas expansões influenciam não só nas condições socioeconômicas e ambientais, mas também na propagação de doenças negligenciadas, pois sua ocorrência pode estar ligada a exposição de pessoas às condições precárias de vida [23,24].

A limitação deste estudo reside, sobretudo, por se tratar de um estudo com dado secundário estando sujeito a subnotificação e má classificação dos casos no banco de dados do SINAN, cujos limites imprecisos poderão ser na identificação do endereço de residência do paciente. Porém a suavização produziu um mapa mais claro da hanseníase em Manaus e os 
resultados obtidos com o alisamento espacial foram tratados com cautela para não mascarar grandes diferenças entre as regiões vizinhas.

O estudo indica a necessidade do fortalecimento da vigilância em saúde em menores de 15 anos no município de Manaus, como ponto crucial para o controle e erradicação da hanseníase nesta população. Conjuntamente o mapeamento da taxa de incidência de hanseníase nos bairros do município foi uma ferramenta válida para a observação das áreas de elevada endemicidade. Esses dados se mostraram preocupantes uma vez que evidenciou a gravidade da situação epidemiológica da hanseníase em Manaus.

O controle da hanseníase em menores de 15 anos em Manaus tem obtido alguns avanços, entretanto seus índices continuam acima da meta proposta pela OMS. A meta a ser seguida está concentrada em zerar os casos novos de hanseníase em menores de 15 anos, o que reflete a importância de maiores esforços dos gestores de saúde do município e do estado para assegurar o acesso à saúde e as medidas de promoção da saúde e prevenção de doenças. Dentro deste contexto, este dado se torna relevante para estabelecer bases para uma ampla discussão no avanço e busca ativa de casos em escolares, a fim de detectar os focos de transmissão ativos em domicilio e iniciar o tratamento, uma vez que a criança é um indicador do nível de transmissão e tem relevância epidemiológica.

Agradecimentos

Este trabalho foi conduzido como parte do Programa Nacional de Cooperação Acadêmica (Procad) / Coordenação de Aperfeiçoamento de Pessoal de Nível Superior (Capes) e com apoio da Fundação de Amparo à Pesquisa do Estado do Amazonas (Fapeam).

Referências

1. World Health Organization. Global Leprosy Strategy: Accelerating towards a leprosyfree world. 2016. [citado 2016 Ago 20]. Disponível em:

http://apps.who.int/iris/bitstream/10665/208824/8/9789290225201-Portuguese.pdf

2. Brasil. Ministério da Saúde. Indicadores epidemiológicos e operacionais de hanseníase Brasil 2001 - 2016. Brasília, 2017. [citado 2017 Set 20]. Disponível em: http://portalsaude.saude.gov.br/images/pdf/2015/outubro/29/S--rie-Hist--rica-Hansen-ase-2000---2014.pdf

3. Talhari S. Hanseníase. 5 ed. Manaus: Dilivros; 2015.

4. Fundação Alfredo da Matta. Boletim Epidemiológico. Manaus, Gerência de IST, ano XVII, n. 24, jan/dez 2016. [citado 2017 Set 10]. Disponível em: https://www.fuam.am.gov.br/wp-content/uploads/2014/05/Boletim 2016.pdf

5. Imbiriba ENB, Silva Neto AL, Souza WV, Pedrosa V, Cunha MGS, Garnelo L. Desigualdade social, crescimento urbano e hanseníase em Manaus: abordagem espacial. Rev Saúde Pública 2009;43(4):656-65. https://doi.org/10.1590/S003489102009005000046

6. Medeiros MS, Sacramento DS, Hurtado-Guerrero JC, Ortiz RA, Fenner ALD. Cost of illness attributable to environmental factors in the city of Manaus, state of Amazonas, Brazil. Ciênc Saúde Coletiva 2014;19(2):599-608. https://doi.org/10.1590/141381232014192.23012012

7. Instituto Brasileiro de Geografia e Estatística. Censo 2010. Brasil, 2010. [citado 2017 Set 10]. Disponível em: http://cod.ibge.gov.br/3WL

8. Brasil. Ministério da Saúde. Resolução 466/12 do Conselho Nacional de Saúde/MS Sobre diretrizes e Normas Regulamentadora de Pesquisa envolvendo seres humanos. 2012. [citado 2017 Set 10]. Disponível em: http://conselho.saude.gov.br/resolucoes/2012/Reso466.pdf

9. Marques REB, Yam Ciribelliaguchi LC, Soares JAS, Fernandes DPC, Alves WA. Morbidade Hospitalar por Hanseníase em Minas Gerais: um estudo epidemiológico no período de 2008 a 2015. J Manag Prim Heal Care 2016;7(1):135.

10. Zanardo TS, Santos SM, Oliveira VCC, Mota RM, Mendonça BOM, Nogueira DS et al. Epidemiological profile of patients with leprosy in primary care of São Luis of Montes 
Belos, the period 2008 2014. Revista Faculdade Montes Belos (FMB) 2014;9(2):77141.

11. Barbosa DRM, Almeida MG, Santos AG. Características epidemiológicas e espaciais da hanseníase no Estado do Maranhão, Brasil, 2001-2012. Medicina (Ribeirão Preto) 2014;47(4):347-56. https://doi.org/10.11606/issn.2176-7262.v47i4p347-356

12. Moreira RLSF, Fontes WD, Barboza TM. Dificuldades de inserção do homem na atenção básica a saúde. Esc Ana Nery Rev Enferm 2014;18(4):615-21. https://doi.org/10.5935/1414-8145.20140087

13. Barreto JG, Bisanzio D, Guimarães LS, Spencer JS, Vazquez-Prokopec GM, Kitron U, Salgado CG. Spatial analysis spotlighting early childhood leprosy transmission in a hyperendemic municipality of the Brazilian Amazon region. PLoS Negl Trop Dis 2014;8(2):e2665. https://doi.org/10.1371/journal.pntd.0002665

14. Freitas BHBM, Cortela DCB, Ferreira SMB. Tendência da hanseníase em menores de 15 anos em Mato Grosso (Brasil), 2001-2013. Rev Saúde Pública 2017;51(28):1-10. https://doi.org/10.1590/s1518-8787.2017051006884

15. Sarmento APA, Pereirao AM, Ribeiro F, Castro JL, Almeida MB, Ramos RM. Perfil epidemiológico da hanseníase no período de 2009 a 2013 no município de Montes Claros (MG). Rev Soc Bras Clín Méd 2015;13(3):180-4.

16. Santino LS, Barreto JÁ, Martins ALGP, Alves FS. Hanseníase dimorfa reacional em criança. Hansen Int 2011;36(1):51-7.

17. Casemiro JP, Fonseca ABC, Secco FVM. Promoting health in school: reflections based on a review of school health in Latin America. Ciênc Saúde Coletiva 2014;19(03):82940. https://doi.org/10.1590/1413-81232014193.00442013

18. Fernandes MVC, Esteves AVF, Castro DB, Rocha EP, Santos CB. Hanseníase na população juvenil e sua relação com a desigualdade social: revisão integrativa. Scientia Amazônia 2017;6(1):117-24.

19. Vieira MCA, Nery JS, Paixão ES, Andrade KVF, Penna GO, Teixeira MG. Leprosy in children under 15 years of age in Brazil: A systematic review of the literature. PLOS Neglected Tropical Diseases 2018;12(10):e0006788. https://doi.org/10.1371/journal.pntd.0006788

20. Souza C, Rodrigues M. Magnitude, tendência e espacialização da hanseníase em menores de 15 anos no estado da Bahia, com enfoque em áreas de risco: um estudo ecológico. Hygeia 2015;11(20):201-12.

21. Simões AL, Freitas CMD. Análise sobre condições de trabalho de Equipe de Saúde da Família, num contexto de vulnerabilidades, Manaus (AM). Saúde em Debate 2016;40(109):47-58. https://doi.org/10.1590/0103-1104201610904

22. Brasil. Ministério da Saúde. Portaria no 3.125, de 7 de outubro de 2010. Brasília, 2010. [citado 2016 Fev 05]. Disponível em: http://www.anvisa.gov.br/ hotsite/talidomida/legis/portaria n 3125 hanseniase 2010.pdf

23. Araújo ES. Desenvolvimento urbano local: o caso da Zona Franca de Manaus. Revista Brasileira de Gestão Urbana 2017;1(1):33-42.

24. Ferreira JEZ, Lima AAM, Silva Junior JA, Mendes RLR. Sistema de informação georrefenciado aplicado ao projeto PROSAMIM - 1/Manaus. Revista de Gestão \& Sustentabilidade Ambiental 2017;5(2):545-58. https://doi.org/10.19177/rgsa.v5e22016545-558 